\title{
Determination of age of Egyptian sphinx located in the front of the pyramids
}

\begin{abstract}
This paper discusses the possibility of Sphinx being older by about a few thousand years. This is because of the weathering signs visible on the Sphinx. To determine the facts, the effects of precession of the earth's axis on weather were considered. It was determined that the precession does not alter the intensity of the weather; it merely shifts the weather towards earlier months of a year. The intensity of weather can change if a place undergoes a change in latitude due to the movement of the earth's plate which takes place over millions of years. Since this possibility does not exist in our case, it is concluded that the age of the Sphinx cannot be moved back to 7000BC as suggested by many researchers.
\end{abstract}

Volume 2 Issue 3 - 2018

\author{
Anand M Sharan \\ Department of Mechanical Engineering, Memorial University of \\ Newfoundland, Canada
}

Correspondence: Anand M Sharan, Department of Mechanical Engineering, Memorial University of Newfoundland, Canada, Email asharan@mun.ca

Received: April 04, 2018 | Published: May 09, 2018

Keywords: calendar, effect of precession of the earth's axis, tropical calendar, weathering by water, change in weather

\section{Introduction}

In recent times, a debate is going on between various archeologists about the age of Sphinx which is in front of great pyramids. ${ }^{1,2}$ It has been a conventional understanding that it is about 4,500 years old, i.e. it dates back to $2500 \mathrm{BC}$. However, some recent researchers believe it is older than $2500 \mathrm{BC}$ more so it was constructed around 7000BC. This new thinking has arisen because as some have claimed that the rounding off of the parts of the Sphinx is due to flow of water around it i.e. Due to large volume of flow of water or water weathering. They believe that such quantities of water flowed around the Sphinx in $7000 \mathrm{BC}$ which is presently in a desert now. They believe that the present day weather around Sphinx changed due to the precession of the earth's axis which has a cycle of approximately 26000 years. However, the traditional archeologists do not agree with this view since there does not exist any evidence of any Egyptian civilization this old. The purpose of this present work is to provide scientific proof that this water erosion belief is not correct.

\section{Motions of earth around the sun and zodiac signs in the three dimensional space}

To understand the change in orientation of the earth's axis due to precession one needs to look at the sky where the zodiacs lie along the zodiac belt. In astrology and historical astronomy, the zodiac signs are distributed along a circle of 12 equal divisions each of $30^{\circ}$ span of celestial longitude that are centered upon the ecliptic. When viewed from the earth it is the apparent path of the Sun as we see during the day across the celestial sphere in the sky. The paths of the Moon and visible planets - also remain close to the ecliptic, within the belt of the zodiac, which extends $8-9^{\circ}$ north or south of the ecliptic, as measured in celestial latitude. The earth goes in its orbit of almost a circular shape around the Sun in about 365.25636 days (Figure 1). In this figure, the distance between the Sun and earth is far smaller than between the Sun and any of the zodiac signs in the zodiac belt During its (earth's) traverse, its north pole is oriented towards the Sun on June 21 and its south pole on December 21 which gives rise to summer and winter seasons respectively. The Moon goes around the zodiac belt in 27.322 days (sidereal) when viewed from the space but 29.531 days (synodic) when viewed from the earth. The earth has a third motion which is called the precession of the earth's axis in the space as shown in Figure 2. Here, this axis rotates as observed in the space along the conical surface once in around 26,000 years which is an extremely slow motion. Due to this motion, the earth's axis changes its orientation in space whereby the North Pole would face the Sun with same orientation on June 20 instead of June 21 after a lapse of about 72 years. Similarly, the equinox will take place on March 20 instead of March 21 after 72 years. In a nut shell, it affects the seasons on the earth by shifting the solstices and equinoxes one day earlier. This precession of the earth's axis only causes a shift in the weather pattern - not any increase or decrease in rain or temperature. The shift in seasons over a long period of time can be seen in Figure 3 where the shift is shown along the arrow. It takes place in the counterclockwise manner. The numbers in this figure show various months of a year. This fact was observed by the European Church and it led to the change in calendar from the Julian to Gregorian as described next., ${ }^{3,4}$

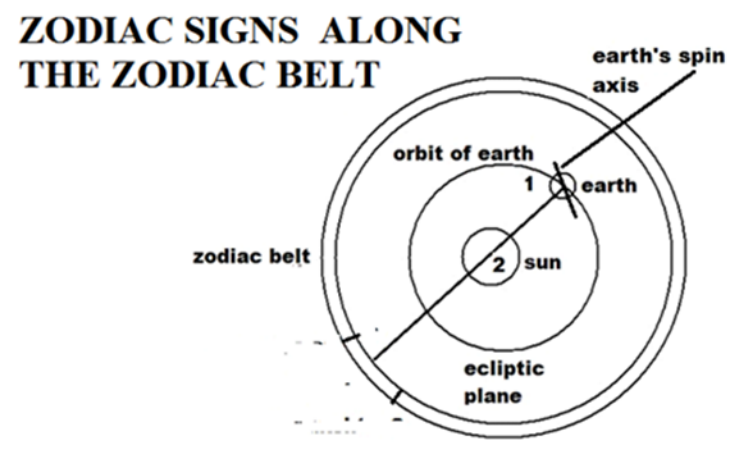

Figure I Zodiac signs along the zodiac belt. 


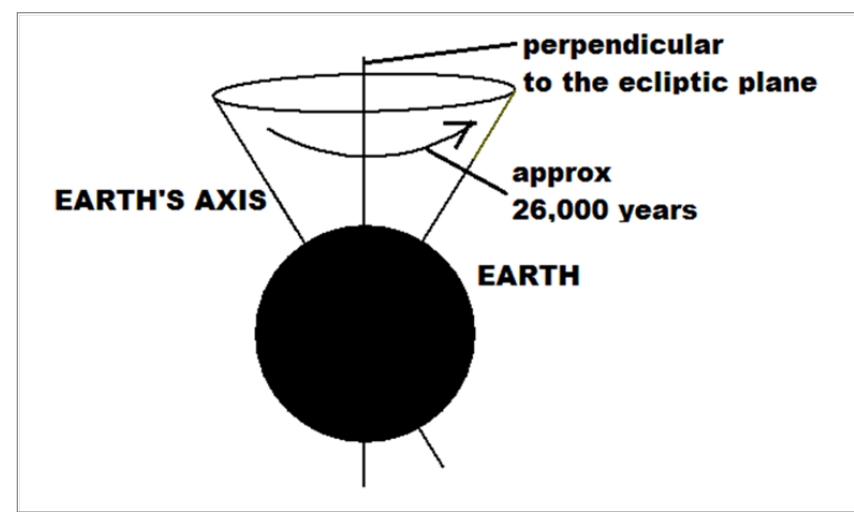

Figure 2 Precession of earth's axis.

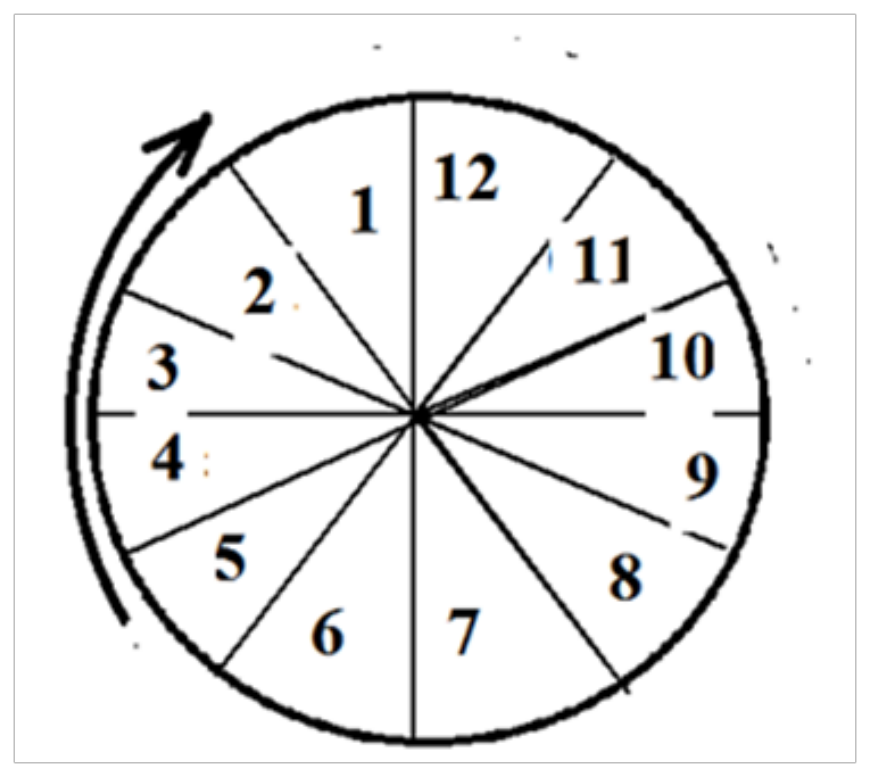

Figure 3 Shifting of season due to precession.

\section{The European (Gregorian) calendar}

The Europeans used Julian calendar prior to 1582 but it was refined in $1582 \mathrm{AD}$ when the length of the year was reduced by about 20 seconds, and this type of year is called a tropical year. ${ }^{5,6}$ It was Pope Gregory who refined the calendar and after 1582 it is called as Gregorian calendar. The reason for the reform was to bring the date for the celebration of Easter to the time of the year in which the First Council of Nicaea held - which was in 325 AD. As mentioned above, in Europe the third type of Motion (precession) was not known and as a result, the seasons (equinoxes) drifted earlier by one day in every 72 years. In $325 \mathrm{AD}$ the spring equinox took place on March 21. In 1582, it had shifted to March $10 .^{3-5}$ Because the celebration of Easter was tied to the spring equinox, the Roman Catholic Church considered this steady drift in the date of Easter undesirable. To stop this drift in the Julian calendar, Pope Gregory reformed the calendar by making the length of the year 20 seconds shorter than a sidereal year observed in the Julian calendar. The consequence of this reform was that the equinoxes after and including 325AD would always occur on
March 21. This shorter year is called a tropical year so that a season on a given day would always remain the same, year after year, but it was a deviation from a sidereal year used in other parts of the world including India.

\section{Water weathering of sphinx due to heavy rain?}

In reference 6 , calculations have been performed to show the regions of the earth where the desert exists. The formation of desert requires long periods of time in millions of years and that it is latitude based. The latitude of sphinx did not change in just thousand years. The change in latitude takes place due to the movement of the earth's plates which take place over millions of years. In Africa this has happened where the Mediterranean Sea has become shorter in area but over millions of years. In other words the latitude of a place can change thereby affecting the rain pattern only when the plate of the earth shifts but this is extremely slow process requiring tens of millions of years. So, the precession of the earth's axis merely causes the seasons to shift earlier but it does not change the intensity of the weather i.e. if around the year there is scanty rain these days around the pyramid then even 7000 or 10,000 years earlier also the total amount of rain would not change. Given this, one can say that the weathering of the Sphinx did not take place due to the flow of water around it as the rainfall in its area is very scarce.

\section{Conclusion}

In the present work, rounding off on the Sphinx in Egypt was examined and the possibility of its construction time earlier than $2500 \mathrm{BC}$ was examined. Since the Sphinx lies in a desert where the rain is scanty, that possibility does not exist since $2500 \mathrm{BC}$. Next, the possibility of excess rain in $7000 \mathrm{BC}$ was examined as indicated by several archeologists. It was scientifically shown that the precession of the earth's axis merely causes shifting earlier of the weather in a calendar year but it does not cause change in the intensity of the seasons. This was illustrated by adoption of change in European Calendar in 1582 by Pope Gregory.

\section{Acknowledgements}

None.

\section{Conflict of interest}

The author declares there is no conflict of interest.

\section{References}

1. MSNBC. How old is the Sphinx?. Mysteries of the Universe. 2013.

2. https://en.wikipedia.org/wiki/Sphinx_water_erosion_hypothesis

3. http://en.wikipedia.org/wiki/Gregorian_calendar

4. http://en.wikipedia.org/wiki/Julian_calendar

5. Snowden B. The Curious History of the Gregorian calendar Eleven Days that Never Were. Sandbox Networks. 2000.

6. Sharan AM. Melting Away of Himalayan Glaciers and Resulting Water Shortages in India and Pakistan. Asian Journal of Water, Environment and

Pollution. 2012;9(3):67-72. 